\title{
Gestão do cuidado à tuberculose: da formação à prática do enfermeiro
}

\author{
Care management for tuberculosis: from education to the nurse practice \\ Gestión del cuidado a la tuberculosis: de la formación a la práctica del enfermero
}

\section{Anne Jaquelyne Roque Barrêto', Anna Luisa Figueiredo Evangelista", Lenilde Duarte de Sá' Sandra Aparecida de Almeida' Jordana de Almeida Nogueira' Ana Maria Cavalcante Lopes'}

' Universidade Federal da Paraíba, Centro de Ciências da Saúde, Departamento de Enfermagem em Saúde Pública e Psiquiatria, Programa de Pós-Graduação em Enfermagem. João Pessoa-PB, Brasil. "Faculdades de Enfermagem e Medicina Nova Esperança, Curso de Graduação em Enfermagem. João Pessoa-PB, Brasil.

Submissão: 03-05-2012 Aprovação: 15-11-2013

\section{RESUMO}

O estudo objetivou analisar a relação entre a formação do enfermeiro e as ações direcionadas à gestão do cuidado à tuberculose. De natureza qualitativa, foi realizada em um dos municípios da região metropolitana de João Pessoa-PB. Participaram do estudo dez enfermeiros da Estratégia Saúde da Família. A coleta de dados foi realizada em fevereiro de 2010 através de um roteiro de entrevista semiestruturado. Para análise do material empírico, utilizou-se a técnica de análise de conteúdo. Nos depoimentos dos enfermeiros há predominância da temática TB no espaço hospitalar, abordando tanto a superficialidade quanto o distanciamento do cuidado à TB no que concerne à relação entre a teoria e a prática, e a ausência de cuidados direto ao doente de TB durante a formação; a organização dos serviços aponta para uma prática mecanicista e tarefeira. Há necessidade das instituições formadoras se aproximarem dos serviços de saúde, numa relação dialógica, para melhor organizar os espaços de aprendizagem.

Descritores: Enfermagem; Atenção Primária em Saúde; Tuberculose.

\section{ABSTRACT}

The study aimed to analyze the relation between the training of the nurse and the actions directed to the management of the care to the tuberculosis. With qualitative nature, it was performed in one of the cities of the metropolitan region of João Pessoa-PB. The subjects were ten nurses of the Health Family Strategy. The data collection was performed in February 2010 using a semi structured interview script. To examine the empiric material it was used the technique of content analyzes. In the testimonies of the nurses there was predominance of the thematic TB in the hospital space; they approach either the superficiality and the distance of the care to TB related to the relation between the theory and the practice, and the absence of direct care to the patient of TB during training; according to them, the organization of the services points to a mechanicist and task based practice. One concluded that there is necessity of training institutions to come close to the health services, in a dialogic relation, to organize better the learning spaces.

Key words: Nursing; Primary Health Attention; Tuberculosis.

\section{RESUMEN}

El estudio objetivó analizar la relación entre la formación del enfermero y las acciones direccionadas a la gestión del cuidado a la tuberculosis. De naturaleza cualitativa, fue realizado en uno de los municipios de la región metropolitana de João PessoaPB. Los sujetos fueron diez enfermeros de la Estrategia Salud de la Familia. La recopilación de datos fue realizada en febrero de 2010 a través de un guión de entrevista semi estructurada. Para análisis del material empírico se utilizó la técnica de análisis de contenido. En las declaraciones de los enfermeros hay predominancia de la temática TB en el espacio hospitalario, en que ellos abordaron tanto la superficialidad como el distanciamiento del cuidado a la TB en lo que se refiere a la relación entre teoría y práctica, y la ausencia de cuidados directos al enfermo de TB durante la formación; la organización de los servicios apunta para una práctica mecanicista y rutinaria. Hay necesidad de que las instituciones formadoras se aproximen de los servicios de salud, en una relación dialógica, para organizar mejor los espacios del aprendizaje.

Palabras clave: Enfermería; Atención Primaria en Salud; Tuberculosis. 


\section{INTRODUÇÃO}

O governo brasileiro, por meio de atos normativos, a exemplo do pacto pela vida, destaca a necessidade do fortalecimento de ações voltadas para gestão do cuidado à tuberculose (TB) com o intuito de garantir o controle efetivo da doença. Segundo a Organização Mundial de Saúde (OMS) ${ }^{(1)}$, estima-se que 100 milhões de pessoas são infectadas anualmente pelo bacilo da Koch em todo mundo. Desses, estima-se, oito a dez milhões desenvolverão a doença durante a vida. A metade dos infectados apresentará a forma contagiante e três milhões deles irão a óbito a cada ano.

No Brasil a TB vem assumindo lugar de destaque entre as prioridades de governo. Políticas de financiamento, bem como a descentralização das ações de controle da TB para as unidades de atenção básica e equipes de saúde da família, ampliaram o escopo de profissionais envolvidos com o diagnóstico, tratamento e acompanhamento dos casos. Tais iniciativas vêm repercutindo na redução da incidência, expressa pela tendência de quedas de novos casos, com média anual de decréscimo de $2,4 \%$. Entretanto, no ranking mundial, o país ainda ocupa a $19^{a}$ posição entre os 22 países responsáveis por $80 \%$ do total de casos de tuberculose. Em 2012, foram notificados no Brasil 70.047 casos novos da doença, com taxas de incidência de 36,1 e mortalidade de 2,4 casos por 100 mil habitantes ${ }^{(2)}$.

Embora seja positiva a trajetória política de ações de controle da TB, estudos assinalam fragilidades no modo de gerenciar, organizar e disponibilizar recursos que facilitem o diagnóstico precoce e produção do cuidado ao doente de TB no âmbito da atenção básica ${ }^{(3-5)}$.

A equipe de saúde da família, por atuar em território definido, exerce um papel determinante para a cura e tratamento da TB. Entretanto, algumas dificuldades no processo de trabalho dessas equipes - como a ineficiência na qualificação profissional para a produção do cuidado ao doente de TB - têm impedido tanto a identificação precoce dos casos, quanto as reais necessidades de saúde dos usuários acometidos.

Como um dos membros da equipe de saúde, o enfermeiro é um ator importante para efetivação do cuidado da TB na medida em que gerencia as ações de controle e compreende a complexidade envolvida nesse processo. No entanto, considera-se necessário que sua formação esteja voltada para o desenvolvimento de habilidades e competências que favoreçam o cuidado integral e humanizado, conforme preconiza o Sistema Único de Saúde (SUS). A formação desses profissionais, no campo da educação superior, deve promover práticas reflexivas como forma de integrar os diferentes tipos de conhecimento para a produção do cuidado das pessoas, especificamente neste estudo, aos doentes de TB. Diante deste contexto questiona-se: a formação do enfermeiro está voltada para promover a gestão do cuidado à TB?

Os apontamentos teórico-conceituais sobre a gestão do cuidado, abordados por Cecílio (2011), nortearão a discussão desse estudo. Compreende-se por gestão do cuidado

o provimento ou a disponibilização das tecnologias de saúde, de acordo com as necessidades singulares de cada pessoa, em diferentes momentos de sua vida, visando seu bem-estar, segurança e autonomia para seguir com uma vida produtiva e feliz ${ }^{(6)}$.

Assim, este estudo tem como objetivo analisar a relação entre a formação do enfermeiro e as ações direcionadas à gestão do cuidado à tuberculose.

\section{MÉTODO}

Estudo de natureza qualitativa, que teve como cenário de estudo, um dos municípios da região metropolitana de João Pessoa-PB. A escolha deste local deu-se por ser considerado prioritário para o controle da TB. Distribuídas em seu território encontram-se dezenove equipes da Estratégia Saúde da Família (ESF), totalizando uma cobertura de $85 \%$.

Participaram do estudo dez enfermeiros, cuja inclusão se deu em função de critérios, como: tempo de atuação na ESF (no mínimo há 01 ano) e presença de casos de TB, devidamente notificados no Sistema de Informação de Agravos de Notificação (SINAN), nas áreas de abrangência da Unidade de Saúde da Família (USF) em que atuam.

Para produção do material empírico utilizou-se a entrevista semiestruturada baseada em um roteiro contendo perguntas subjetivas relacionadas à formação do enfermeiro e a prática da gestão do cuidado à TB. A coleta de informações foi realizada no mês de fevereiro de 2010. Cada entrevistado recebeu informações sobre a natureza e os objetivos do estudo, assim como a permissão para participação mediante assinatura do consentimento informado. As entrevistas foram realizadas nas USF, local de trabalho dos sujeitos, de acordo com a disponibilidade de horário por eles informada.

Os depoimentos foram gravados mediante aquiescência dos sujeitos e posteriormente transcritos. Para o tratamento dos dados empíricos, optou-se pela utilização da Técnica de Análise de Conteúdo Categorial, por ser entendida como um meio de expressão do sujeito. Por meio dessa técnica o analista categoriza palavras ou frases que aparecem com mais frequência no texto e, posteriormente, inferindo uma expressão que possa representá-los de forma adequada ${ }^{(7)}$. Nesse sentido, as transcrições foram lidas repetidas vezes e, a partir desta leitura, foram construídas duas categorias: A formação do enfermeiro para gestão do cuidado à TB e Prática do enfermeiro na gestão do cuidado à TB.

Para facilitar a apresentação dos resultados e manter o sigilo quanto à identidade dos sujeitos, foi atribuído um número seqüencial a cada participante (E1, E2, E3... E10). Em todas as etapas deste estudo foram respeitados os aspectos éticos da Resolução 196/96 CNS/MS que dispõe sobre o envolvimento direto ou indireto com seres humanos em pesquisa bem como a Resolução no 311/2007 COFEN que aprova o Código de Ética dos profissionais de enfermagem. Sua aprovação se deu pelo Comitê de Ética em Pesquisa da Faculdade de Enfermagem Nova Esperança, sob o $n^{\circ}$ do Protocolo 09/2010 e CAEE: 0176.0.000.351-10.

\section{RESULTADOS E DISCUSSÃO}

A formação do enfermeiro para gestão do cuidado à TB 
Os depoimentos dos enfermeiros revelam que no curso de graduação os espaços de aprendizagem estavam voltados, principalmente, para o campo curativo. Observa-se que os sujeitos com maior tempo de formação tiveram sua experiência para o controle da doença, exclusivamente no hospital de referência do estado. Já os enfermeiros com menos de dez anos de formação, além do espaço hospitalar, também vivenciaram o cuidado à TB na Unidade de Saúde da Família (USF).

Passamos também pelo Clementino, [...]. Na época também passamos pelo pronto socorro Municipal. Os estágios geralmente eram no HU mesmo. [E4]

Somente nos estágios curriculares que tivemos [experiência] no Clementino Fraga. [E6]

A gente pagou a disciplina e na prática fomos para o Clementino Fraga. [E8]

O que chama atenção nos depoimentos dos enfermeiros relaciona-se à predominância da temática TB no espaço hospitalar, o que favorece a formação de profissionais com olhar restrito à clínica da doença. A experiência dos enfermeiros, colaboradores do estudo, no processo de sua formação evidenciam um aparente distanciamento entre o que rege a política de formação de trabalhadores para o SUS e o ensino.

A Resolução $n^{\circ} 3$ do Conselho Nacional de Educação(8) institui as diretrizes curriculares do curso de enfermagem, as quais orientam a formação do enfermeiro para atuar nos distintos níveis de atenção em saúde. A formação do enfermeiro deve atender as necessidades sociais da saúde, com ênfase no Sistema Único de Saúde, e assegurar a integralidade da atenção e a qualidade e humanização do atendimento.

Observa-se nas falas dos enfermeiros que às matrizes curriculares enfatizam uma abordagem estritamente biológica colaborando para formação de profissionais com agir em saúde puramente clínico e, consequentemente, distanciado da realidade em que os grupos populacionais vivenciam o adoecer de TB.

No período que eu estava cursando Enfermagem, foi muito simples, porque como a gente estudava doenças infectocontagiosas vimos só uma parte. Então, não foi muito aprofundado para o controle, foi mais aprofundado a questão da assistência. [E3]

Somente o que a gente aprendeu em sala de aula [os sinais e sintomas da TB], porque a teoria é totalmente diferente da prática. [E2]

Na graduação a gente não vê isso [controle da tuberculose] muito detalhado, quando a gente veio pra estágio em PSF foi que vi melhor essa parte [de controle]. [E9]

Percebe-se pelas falas que os enfermeiros abordaram tanto a superficialidade quanto o distanciamento do cuidado à TB no que concerne à relação entre a teoria e a prática. Pressupõe que a inadequação dos processos de ensino-aprendizagem tradicionais e organizados em disciplinas concorre para manter lacunas existentes entre instituição formadora e dinâmica do serviço. Quando se trata de uma doença social e estigmatizada, considera-se importante que esse profissional não aprenda apenas a clínica, tratamento e controle, mas que avance na compreensão da subjetividade e singularidade envolvidas no processo de cuidar pautado, sobretudo, na integralidade do cuidado.

A intrigante tensão teoria/prática permeia os mais diversos setores da sociedade. Um exemplo para tal dicotomia é o que acontece em grande parte dos cursos de formação dos profissionais de saúde, nos quais, muitas vezes, a teoria aparece dissociada da prática, não dando margem à reflexão acerca da relação existente entre elas ${ }^{(9)}$, o que impede o profissional de pensar sobre o plano de cuidado específico para cada usuário. Talvez um dos grandes entraves para tal dicotomia esteja relacionado ao entendimento e/ou vivência de políticas públicas de cunho preventivo que prevaleceram desde o início do século passado.

É necessário que as matrizes curriculares das instituições de ensino superior favoreçam a formação do profissional de saúde para atuar, não somente na atenção aos usuários do SUS, mas acima de tudo, que essa assistência seja feita sob o uso de tecnociências em consonância com os princípios do SUS e segundo a concepção da Atenção Primária em Saúde (APS). Para tanto é necessário utilizar abordagens pedagógicas que possibilitem uma maior aproximação entre o que é visto na teoria com o que é vivenciado na prática. Os processos pedagógicos devem aliar métodos que possam favorecer a construção de profissionais críticos e reflexivos e que, sobretudo, compreendam o cuidado como valor.

O cuidado como valor nasce no entendimento de ultrapassar as fragmentações de saberes existente no campo da saúde. Defende a reintegração entre o agir e o pensar em saúde, enquanto agir político e valor ético na construção de práticas eficazes de integralidade ${ }^{(10)}$.

Reconhece-se que as metodologias ativas de aprendizagem sejam viáveis nessa proposta, pois o estudante se coloca ao lado do professor que tem a tarefa de orientar e facilitar o processo educativo como um ser que também busca o conhecimento. Entretanto, dialogar com esses estudantes não significa Ihes delegar toda a elaboração do problema, mas sim conduzi-los a um processo que possibilite uma análise profunda da problemática, a fim de que possam descobrir sua dimensão e buscar respostas às questões propostas ${ }^{(11)}$.

Ao ampliar essa discussão, há necessidade de compreender que a construção de um pensamento fundado no reconhecimento da transdisciplinaridade colabora na conformação de profissionais atuantes numa perspectiva de trabalho em equipe ${ }^{(12)} \mathrm{e}$, consequentemente, com à gestão do cuidado. Nesse sentido, os métodos pedagógicos, utilizados pelas instituições formadoras, devem auxiliar na formação de profissionais de saúde com habilidades de enxergar o usuário como sujeito singular, com vivências e histórias a serem consideradas no processo de cuidar, exercendo assim, sua capacidade autopoiética, ou seja, a autocriação contínua. A relação entre viver, conhecer e fazer coloca a vida como criação e o conhecer como ato de poiesis (recriação) ${ }^{(13)}$.

Assim, se faz necessário apostar em abordagem que concilie a filosofia e a política. Estes conteúdos quando articulados, 
possibilitam "diálogos epistemológicos produtivos e necessários ao desenvolvimento da capacidade humana de valorar e constituir seu ethos" - entendido aqui como o ser humano organiza sua vida, seja nas particularidades da esfera privada, seja nas singularidades produzidas no grupo - principalmente na sua maneira de conhecê-los e analisa-los ${ }^{(10)}$.

Outro ponto identificado nas falas dos enfermeiros refere-se ao não acompanhamento de pessoas doentes de TB nos espaços de estágio, havendo muitas das vezes, apenas, a apresentação da estrutura e da organização do serviço de saúde e observação da assistência ao doente.

A gente via a teoria e depois víamos a parte prática. É tanto que quando fomos para prática não tivemos oportunidade de acompanhar um paciente com TB. [E4]

Vi a parte da assistência no Clementino, à parte ambulatorial e de internação, mas não acompanhamos nenhum paciente. [E10]

A deficiência na formação, no que se refere aos cuidados direto, é verbalizada nas falas acima, com ênfase na "observação" de cuidados prestados aos doentes pelos profissionais do hospital. Além disso, não há depoimentos, dos enfermeiros entrevistados que ressalte os cuidados preventivos, nem tampouco mencionam que a sua realização é feita priorizando a família e comunidade, já que são constituintes da vida dos doentes.

A falta de contato direto do estudante de enfermagem com a pessoa doente de TB impossibilita o reconhecimento do ethos cultural de quem é cuidado. Segundo Pinheiro ${ }^{(10)}$, se o ethos for compreendido como uma "dimensão prática da vida humana", se observará, que sua irá auxiliar o ser humano no reconhecimento da diversidade/pluralidade expressas por demandas, compreensíveis, muitas vezes, por saberes produzidos localmente. Nessa dinâmica se faz necessário que o estudante de enfermagem compreenda particularidades culturais e singularidades das pessoas doentes de TB, tendo em vista ser uma doença de cunho social.

O estabelecimento de vínculo entre os profissionais e usuários na assistência é de grande importância para sua formação, pois além de permitir uma melhor sensibilização, ajuda o profissional a relacionar-se com a prática cotidiana identificando as reais necessidades desse usuário.

Reconhece-se que a aprendizagem do cuidado de enfermagem poderia ser melhor desenvolvida no contato direto do aluno com o usuário do serviço de saúde porque proporciona ao discente a possibilidade de vivenciar diferentes contextos na prática, ao qual favorece o estabelecimento das relações de cuidado nos distintos espaços de aprendizagem ${ }^{(14)}$. A esse respeito, Bousso enfatiza que o campo de estágio "é o lócus onde a identidade profissional do aluno é gerada, construída e referida; volta-se para o desenvolvimento de uma ação vivenciada, reflexiva e crítica"(15).

Importante ressaltar que quando indagados acerca da influência da formação na dificuldade de controle dos casos de TB, inclusive nos casos de abandono, alguns enfermeiros mencionaram que não há relação, pois depende da responsabilidade e compromisso do profissional em buscar a qualificação, considerando a aprendizagem em seu lócus operandi, isto é, a partir do seu espaço de atuação e reflexão sobre as práticas de cuidado.

Eu acho que vai muito do interesse do profissional, porque a academia que a gente exerce vale muito do estudante. [E1]

[...] não é determinado pelo que a gente aprende na faculdade, mas sim pelo que o profissional faz por esse paciente, então depende muito da vontade que o profissional tem. [...] O profissional ele tem que se envolver, tem que fazer com que o paciente acredite no que você está colocando para ele. [E9]

Entretanto, outros depoimentos mencionaram que a maneira como as instituições formadoras ensinam sobre TB influenciam na efetividade da gestão do cuidado, tanto nas dimensões individual, quanto organizacional.

Acho que pode influenciar sim, pois assim que uma pessoa termina o curso, inicia o seu trabalho e pega um caso desses (de tuberculose), se ele não tiver tido uma experiência ou uma sensibilização maior para a tuberculose, ele não conseguirá dar conta da prática. [E6]

Eu acho que influencia, porque você pode até não ter se aprofundado naquele assunto, mas se a pessoa pagou a disciplina bem, assistiu às aulas e teve interesse sobre o assunto, aí sim, quando chegar à prática você vai só aplicar o que aprendeu. Como é que você vai tratar um paciente se você não teve uma boa formação? [E8]

Faz-se necessário esclarecer que a educação superior vem sendo desafiada a romper com modelos tradicionais de ensino - que favorecem a fragmentação de saberes e práticas em detrimento a transdisciplinaridade - para direcionar uma formação com pertinência social e coerência com as Diretrizes Curriculares Nacionais (DCN), ou seja, com as necessidades de transformação do processo de formação profissional.

O novo modelo de atenção construído a partir da concepção da APS, no Brasil representado pela Estratégia Saúde da Família, exige novas formas de construção do conhecimento, pressionando mudanças no processo de formação de profissionais capacitados para o atendimento da população. Essa necessidade de mudança decorre de elementos, tais como as novas modalidades de organização do mundo do trabalho em saúde e das exigências no perfil de novos profissionais voltados para a transdisciplinaridade na produção do conhecimento ${ }^{(16)}$.

Salienta-se que, entre as atribuições das instituições formadoras, está a responsabilidade em construir pensamentos reflexivos e críticos sobre os fenômenos estudados/vivenciados, inclusive a capacidade de instigar no sujeito, neste caso o estudante de enfermagem, o aprendizado baseado nos quatro pilares da educação moderna que são: aprender a aprender; aprender a fazer; aprender a conviver e aprender a ser. Por outro lado, se faz igualmente necessário que este profissional busque a qualificação/atualização perene para que venha 
compreender a dinamicidade dos problemas advindos da realidade local para a posteriori operar processos que venham resolver/minimizar tais adversidades.

O fato de o profissional de saúde referir que não há influência da formação com a gestão do controle da TB, não exclui a responsabilidade da instituição na preparação de profissionais que venham dar respostas para o sistema e que contribuam efetivamente para a gestão do cuidado à TB, em suas distintas dimensões, especificamente na profissional que se encontra diretamente relacionada à formação desse trabalhador nas instituições de ensino(6).

Desse modo, uma política educacional deverá considerar a reintegração entre o pensar e o agir em saúde, de modo que favoreça a formação de profissionais baseada na valoração - dar sentido à sua existência a fim de optar entre várias possibilidades de ser - na responsabilização e na ética ${ }^{(10)}$. Nesta perspectiva os processos de formação, poderia possibilitar uma efetiva articulação entre universidade e serviços/sistema de saúde. O processo de aproximação e construção de compromissos, a responsabilidade pública e a relevância social da universidade, serão ampliados na medida em que se traga para o contexto da educação o conjunto das diretrizes do SUS, em especial a integralidade ${ }^{(17)}$, compreendida como "dispositivo legal-institucional, portador de valores ético-políticos, que têm no cuidado a sua maior expressão como atividade humana"(10).

\section{Prática do enfermeiro na gestão do cuidado à TB}

Sobre a organização do serviço para a gestão do cuidado à TB os depoimentos dos enfermeiros apontam para uma prática mecanicista e tarefeira mais coerente com a concepção biomédica, onde a partir dos sinais e sintomas apresentados pelo doente elaboram o menu da assistência como atividades relacionadas ao diagnóstico, tratamento e monitoramento.

[Eu] realizo uma anamnese nele [pessoa com suspeita de TB], pedimos uma baciloscopia com duas amostras de escarro, mediante o resultado dessa baciloscopia, a gente começa o tratamento [...] e seque o esquema [de tratamento contra TB] do Ministério da Saúde e faz o acompanhamento [da pessoa com diagnóstico de TB]. [E1]

O paciente chega com tosse há mais de três semanas, pedimos o exame de baciloscopia e ele leva para o laboratório. [...] Sendo confirmado o diagnóstico ele vai até a unidade para receber a medicação. Todo mês o usuário vem pegar a dosagem, daí a gente pesa para saber se eles estão se alimentando direito. [E2]

Em contrapartida, outros depoimentos direcionam uma organização de serviço para a atenção à TB voltado a busca ativa de sintomáticos respiratórios, esta, especialmente realizada pelo Agente Comunitário de Saúde (ACS).

Alguns pacientes detectados que estão com TB procuram a unidade e outras vezes, é necessário um estado de busca ativa. Às vezes [...] um ACS vem até a gente com paciente suspeito (de TB), daí fazemos a visita domiciliar. [E3]
Através da busca ativa com o agente (ACS) de saúde que faz o elo entre a comunidade e a gente. A gente busca o sintomático respiratório, solicita a baciloscopia, daí dependendo do resultado é feito o controle e o tratamento na unidade. [E7]

Observa-se que a organização do serviço se volta para a detecção do sintomático respiratório (SR), principalmente a busca passiva de casos; diagnóstico e monitoramento do doente de TB. Não foram identificadas nas falas ações mais ampliadas que favorecesse a construção de autonomia e ao desenvolvimento de habilidades pessoais tão importantes na luta contra a TB. A manutenção de equipamentos e insumos, da mesma forma que a garantia de acesso nos diversos níveis de complexidade. A identificação de pessoas suspeitas e/ou com diagnóstico de TB é importante, mas é limitada quando o olhar do profissional se encontra pautado em concepções biomédicas e curativas do cuidado o que reflete, algumas vezes, ao tipo de experiência vivenciada no âmbito da graduação.

Neste enfoque, o modo como se organizam os serviços de saúde desperta uma série de reflexões acerca da assistência prestada. Onde já existe oferta de serviços de saúde organizados na lógica da atenção médica curativa, a introdução de novas estratégias de trabalho implica repensar o modelo de assistência, remodelar o "velho" e construir o "novo" mediante a constituição do cuidado como um agir político e valor ético na construção de práticas eficazes de integralidade ${ }^{(10)}$. Nem sempre esta tarefa é fácil, pois exige da equipe uma desconstrução do trabalho produzido e disposição para o "fazer" diferente, em um cenário extra USF que utiliza outros instrumentos de trabalho, outros saberes e formas de comunicação com a população ${ }^{(18)}$. Sendo tarefa difícil quando na prática depara-se com profissionais de saúde engessados em concepções hegemônicas e que são referenciadas deste sua formação.

Apenas um enfermeiro, em seu depoimento, relacionou ações educativas como atividade que favorece a detecção precoce de casos suspeitos de TB.

A gente realiza atividades educativas que a gente faz na comunidade, tem o dia $D$ aonde fazemos o movimento, a atuação dos ACS fazendo a captação precoce, durante as consultas os pacientes que tem a sintomatologia [...] a gente solicita os exames para investigar o diagnóstico. [E5]

As ações de educação em saúde é uma atividade essencial para que a comunidade reconheça a sintomatologia da TB, sobretudo para favorecer a ruptura de medos e preconceitos da população com a doença. É de responsabilidade da equipe de SF o desenvolvimento de ações educativas junto aos usuários na unidade de saúde, bem como na própria comunidade ${ }^{(19)}$.

Destarte, as ações de educação em saúde precisam ser reconhecidas como alicerce da Atenção Básica e inseridas no âmbito da Estratégia Saúde da Família com a finalidade de promover uma melhor apreensão dos usuários acerca do processo saúde e doença e, consequentemente, ofertar subsídios para adoção de novos hábitos de vida e medidas de saúde, ou seja, contribuir efetivamente para emancipação de sujeitos $^{(20)}$. O enfermeiro ao reconhecer a educação em saúde 
como estratégia para detecção precoce de SR revela que há um processo de construção de ações voltadas para o campo da vigilância da saúde o que demonstra que, embora na época de sua formação, esse conceito não era trabalhado, o enfermeiro prossegue no conceito de controle, aprendido em sua formação para o de vigilância em saúde.

A utilização do tratamento diretamente observado (TDO) foi mencionada, por apenas três enfermeiros, como estratégia para acompanhamento do doente de TB, entretanto, apenas referem-se à visita do ACS para averiguar a tomada da medicação. Importante ressaltar que o TDO é uma parte do DOTS, o que deixa claro a ausência ou ineficiência da formação do enfermeiro em uma política mais ampla que a simples verificação da tomada dos remédios.

[...] quando é confirmado o diagnóstico o paciente é tratado aqui mesmo na unidade utilizando o DOTs, daí a gente fica acompanhando e fazendo visita domiciliar para saber se ele está tomando a medicação correta. [E4]

Notificando o caso ele começa o tratamento e toma a dose supervisionada aqui e toda semana o agente (comunitário) de saúde vai fazer a visita para saber se ele está realizando o tratamento correto. [E9]

Contudo, os enfermeiros ao relacionar o TDO apenas com o monitoramento da tomada do medicamento fragiliza a construção de um cuidado integral à pessoa doente de TB, conforme preconiza o SUS, e fortalece, além da exclusão social, uma organização de serviços e de atenção centrada na cura que revela uma visão biologicista.

\section{CONSIDERAÇÕES FINAIS}

Considera-se que a formação experienciada pelos enfermeiros, colaboradores deste estudo, não foi favorável para a produção do cuidado à TB. Há necessidade das instituições formadoras se aproximarem dos serviços de saúde, numa relação dialógica, para melhor organizar os espaços de aprendizagem, ao mesmo tempo em que, as metodologias adotadas nesse processo sejam capazes de construir sujeitos críticos e reflexivos sobre a realidade a ser enfrentada.

Para gestão do cuidado à tuberculose ser efetiva requer a formação de profissionais orientados à concepções que orientem a compreensão da saúde como direito social, a exemplo da determinação social da doença, bem como as práticas de cuidado em saúde sejam operacionalizadas por meio da ética, da responsabilização e do acolhimento.

Reconhece-se que ainda há um longo percurso a ser trilhado na perspectiva da formação de profissionais, enfermeiros, que atuem com práticas inovadoras de cuidado e que se aproximem aos preceitos do SUS, não enquanto sistema de saúde, mas como uma política que vislumbre à saúde enquanto um direito e bem social.

\section{REFERÊNCIAS}

1. World Health Organization. Global tuberculosis report. Geneva: WHO; 2012.

2. Ministério da Saúde. Secretaria de Vigilância em Saúde. Tuberculose: alinhada com o social, afinada com a tecnologia. Bol Epidemiol [periódico na internet]. 2013 [acesso em 19 nov 2013];44(2). Disponível em: http://portalsaude.saude.gov.br/portalsaude/arquivos/pdf/2013/Abr/10/ boletim2 2013 tb web.pdf

3. Figueiredo TMRM, Villa TCS, Scatena LM, Gonzales RIC, Ruffino-Netto A, Nogueira JA, et al. Desempenho da atenção básica no controle da tuberculose. Rev Saúde Pública. 2009;43(5):825-31.

4. Sá LD, Gomes ALC, Nogueira JA, Villa TCS, Souza KMJ, Palha PF. Intersetorialidade e vínculo no controle da tuberculose na Saúde da Família. Rev Latino-Am Enferm. 2011;19(2):[09 telas].

5. Nogueira JA, Silva CA, Trigueiro DRSG, Trigueiro JVS, AImeida SA, Sá LD, et al. A formação de profissionais de saúde na atenção à tuberculose: desafios e contradições da prática. Rev Enferm UFPE on line. 2011;5(4):778-87.

6. Cecílio LCO. Apontamentos teórico-conceituais sobre processos avaliativos considerando as múltiplas dimensões da gestão do cuidado em saúde. Interface 2011;15(37):589-99.

7. Bardin L. Análise de Conteúdo. Lisboa: Edições 70; 2004.
8. Ministério da Educação (Brasil). Resolução $n^{\circ}$. 03, de 07 de novembro de 2001. Institui diretrizes curriculares nacionais do curso de graduação em enfermagem. Diário Oficial da união 09 nov 2001; Seção 1.

9. Melo TMM. A conjugação teoria/prática na sala de aula comunicativa de ILE: uma experiência de pesquisa-ação. Linguagem \& Ensino. 2002; 5(1):149-69.

10. Pinheiro R. Cuidado como um valor: um ensaio sobre o (re)pensar e a ação na construção de práticas eficazes de integralidade em saúde. In.: Pinheiro R, Mattos RA, organizadores. Razões públicas para a integralidade em saúde: o cuidado como valor. Rio de Janeiro: ABRASCO; 2009. p.15-28.

11. Campos MAF, Forster AC. Percepção e avaliação dos alunos do curso de medicina de uma escola médica pública sobre a importância do estágio em saúde da família na sua formação. Rev Bras Educ Méd. 2010;32(1):83-89.

12. Pinheiro R, Barros MEB, Mattos RA. Trabalho em equipe: a estratégia teórica e metodológica da pesquisa sobre integralidade em saúde. In: Pinheiro R, Barros MEB, Mattos RA, organizadores. Trabalho em equipe sob o eixo da integralidade: valores, saberes e práticas. Rio de Janeiro: ABRASCO; 2010. p.19-36.

13. Ceccim RB, Guimarães AR, Klafke TE, Lenz FL. Autogestão no trabalho com/em equipes de saúde: estudantes agindo o Sistema Único de Saúde. In: Pinheiro R, Barros 
MEB, Mattos RA, organizadores. Trabalho em equipe sob o eixo da integralidade: valores, saberes e práticas. Rio de Janeiro: ABRASCO; 2010. p. 179-204.

14. Guedes GF, Ohara CVS, Silva GTR, Franco RRM. Ensino clínico na enfermagem: a trajetória da produção científica. Rev Bras Enferm. 2009;62(2):283-6.

15. Bousso RS, Merighi MAB, Rolim MA, Riesco MLG, Angelo M. Estágio curricular em enfermagem: transição de identidades. Rev Esc Enferm USP. 2010;34(2):218-25.

16. Lopes WO, Saupe R, Massaroli A. Visita domiciliar: tecnologia para o cuidado, o ensino e a pesquisa. Ciênc Cuid Saúde. 2008;7(2):241-7.

17. Gonzáles AD, Almeida MJ. Integralidade da saúde
- norteando mudanças na graduação dos novos profissionais. Ciênc Saúde Coletiva. 2010;15(3):757-62.

18. Muniz JN, Palha PF, Monroe AA, Gonzales RC, Ruffino Netto, Villa TCS. A incorporação da busca ativa de sintomáticos respiratórios para o controle da tuberculose na prática do agente comunitário de saúde. Cienc Saúde Coletiva. 2005;10(2):315-21.

19. Ministério da Saúde. Princípios e Diretrizes para a NOB/ RH-SUS. 2. ed. Brasília (DF): Ministério da Saúde; 2000.

20. Trigueiro JS, Silva ACO, Gois GAS, Almeida SA, Nogueira JA, Sá LD. Percepção de enfermeiros sobre educação em saúde no controle da tuberculose. Cienc Cuid Saúde. 2009:8(4):660-6. 